\title{
Development and validation of a questionnaire to self-assess patient knowledge of direct oral anticoagulants (KODOA-test)
}

This article was published in the following Dove Press journal: Drug, Healthcare and Patient Safety

\section{Corina Metaxas' \\ Valerie Albert ${ }^{1}$ \\ Matthias Stahl ${ }^{2}$ \\ Kurt E Hersberger' \\ Isabelle Arnet' \\ 'Department of Pharmaceutical Sciences, Pharmaceutical Care Research Group, University of Basel, Basel, Switzerland; ${ }^{2}$ Department of Internal Medicine, Kantonsspital Basel-Land, Bruderholz, Switzerland}

Correspondence: Corina Metaxas Pharmaceutical Care Research Group, Pharmazentrum, Klingelbergstrasse 50, $\mathrm{CH}-4056$ Basel, Switzerland

$\mathrm{Tel}+4$ I 6I 207 I944

Email corina.metaxas@unibas.ch
Objective: Few studies have examined associations between patient knowledge of direct oral anticoagulants (DOAC) and clinical outcomes, mostly because of the lack of validated questionnaires for assessing knowledge. The aim of this study was to develop and validate a questionnaire to self-assess knowledge of DOAC.

Methods: Twelve anticoagulation experts participated in the questionnaire development process to ensure content validity. The Knowledge Of Direct Oral Anticoagulants (KODOA)-test was submitted to patients on DOAC and to pharmacists to assess construct validity. Responsiveness was evaluated after educational counseling. Test-retest reliability was assessed to ensure stability over time, and Cronbach's $\alpha$ was calculated for internal reliability. Index of difficulty and item discrimination ( $D$-value) were calculated to assess the performance of single items.

Results: The KODOA-test contains 15 items with multiple-choice answers. Each correct answer scores 1 point (max. score of 15). The KODOA-test was administered to 32 patients on DOAC and 28 pharmacists. Pharmacists scored significantly higher than patients at baseline (median score 13.3 vs $10.0 ; p<0.001)$, supporting construct validity. Patient scores increased significantly after educational counseling (median score 11 [interquartile range 2] vs 14 [interquartile range 3]; $p<0.001)$. Test-retest and Cronbach's $\alpha$ were acceptable with a Pearson's correlation of 0.8 and an $\alpha$ of 0.67 . The index of difficulty for most items was satisfactory (0.38-0.72) and the mean $D$-value was $42.5 \%$.

Conclusion: The KODOA-test is a brief, valid, and reliable knowledge self-assessment questionnaire that may be used in clinical trials to investigate associations between knowledge increase and patient-related outcomes.

Keywords: patient knowledge, direct oral anticoagulants, questionnaire development, adherence, validation

\section{Introduction}

After a 50 years use of vitamin $\mathrm{K}$ antagonists (VKAs) as oral anticoagulant agents (OACs), a new class of substances with different mechanism of action, the direct oral anticoagulants (DOAC), also called non-vitamin $\mathrm{K}$ oral anticoagulants, has been developed. DOAC are recommended for the prevention and treatment of thromboembolic diseases such as for the long-term prevention or treatment of thrombosis ${ }^{1}$ and for the prophylaxis of stroke and systemic embolism in nonvalvular atrial fibrillation (AF). ${ }^{2}$ Controlling blood values is needed to maintain appropriate anticoagulation with VKAs due to the narrow therapeutic window, which makes the treatment challenging. In contrast to VKA, DOAC do not require routine monitoring, have a fixed dose 
regimen, and possess no restriction on dietary consumption of vitamin K-containing food. Therefore, DOAC seem more convenient for patients than therapy with VKAs. In practice, use of DOAC to treat AF is increasing. ${ }^{3,4}$ However, due to their short half-life, DOAC's anticoagulant effect is likely to be rapidly reduced after the omission of one dose when no counterbalancing action is undertaken. ${ }^{5}$ Thus, DOAC's daily intake requires a strict timing adherence to ensure appropriate therapeutic coverage. Because DOAC do not require consultations for monitoring, the opportunity to discuss aspects of adherence with the patient during such encounters will be missing. Consequently, other ways of insuring adherence to DOAC are required.

Improvement of knowledge and adherence in patients on OACs, in particular DOAC, is needed. ${ }^{6}$ For VKAs, enhanced patient knowledge of OACs and the underlying disease was associated with improved long-term adherence or better therapeutic outcomes. ${ }^{7-9}$ To study associations between knowledge and adherence, validated questionnaires are needed to assess patient knowledge of DOAC. To date, various questionnaires exist that assess OAC knowledge. However, only a few have been psychometrically validated, such as the Oral Anticoagulation Knowledge (OAK), ${ }^{10}$ the Anticoagulation Knowledge Assessment (AKA) test ${ }^{11}$ and the recently published Anticoagulation Knowledge Tool (AKT). ${ }^{12}$ Only AKT can be used for VKAs and DOAC. To our knowledge, there is no specific knowledge assessment questionnaire for DOAC available.

The objective of this study was to develop and validate a questionnaire to self-assess patient knowledge of DOAC.

\section{Methods}

\section{Trial design}

The study was initiated by the Pharmaceutical Care Research Group of the University of Basel. The study was conducted in accordance with the Declaration of Helsinki and has been registered at ClinicalTrials.gov ID NCT03124654. The study was approved by the regional ethic committee (Ethikkommission Nordwest- und Zentralschweiz UBE15/126). All patients gave written informed consent.

\section{Development of the Knowledge Of Direct Oral Anticoagulants (KODOA)- test}

\section{Literature search}

A systematic literature search in Medline and Embase was conducted in March 2015 to retrieve published questions assessing patient knowledge of anticoagulation treat- ment. The search strategy was performed with truncated terms: "Patient* education*" AND "Anticoagulant*" OR "Anticoagulation treatment*" OR "Knowledge" and the limits: English language, human, published from 2005 to march 2015. Abstracts were screened and full texts were retrieved. Two authors (CM and VA) extracted the items of interest. Items regarding VKAs were adapted to DOAC if applicable. Consensus was reached by discussion. Swiss summaries of Product Characteristics, the Updated European Heart Rhythm Association (EHRA) Practical Guide on the use of non-vitamin $\mathrm{K}$ antagonist anticoagulants, ${ }^{13}$ the patient guide for taking DOAC from a cardiology patient page ${ }^{14}$ and patient information leaflets of the 4 in Switzerland authorized DOAC were used to retrieve additional content. Items were grouped into the 9 educational topics as follows: ${ }^{15}$ 1) underlying disease; 2) risk-benefit of treatment; 3) mode of action; 4) application and treatment adherence; 5) accessing health care providers; 6) relevant blood tests; 7) medication interactions; 8) diet and lifestyle; and 9) self-care.

\section{Item reduction and content validity}

A panel of experts was created with 12 health care professionals (HCP; 4 nurses, 4 pharmacists, and 4 physicians) who had experience with DOAC patients. They selected the relevant items for patient knowledge of DOAC and determined the extent to which the items represent the construct of interest (content validity). ${ }^{16}$

The item reduction occurred with an online survey ( 2 rounds) followed by a focus group discussion. The 9 educational topics (first round) and the single items (second round) were ranked in descending order (first = most relevant topic, last $=$ most irrelevant topic). The focus group discussion aimed at defining which items had to be included in the questionnaire by defining a threshold above which the items were perceived as relevant for patient knowledge. Discussion and setting threshold occurred by voting until a consensus was found (unanimity). The session was recorded and transcribed verbatim.

\section{Answer format}

Answers to the items were developed as multiple-choice format because this is the most appropriate and efficient form for assessing cognitive knowledge. ${ }^{17}$ One correct and 2 incorrect response alternatives were adapted from the original article when available or were newly created. A score was defined as 1 for a correct response and 0 for an incorrect or no response. Confidence about the given response was assessed with a single question with a yes/no answer option. 


\section{Educational manual with correct answers as counseling guide}

A manual with referenced correct answers and background information about the reason for correctness was generated in order to standardize the education provided to patients. The manual is not supposed to be given to patients. The manual starts with an instruction section about how to dispense the educational counseling. The HCP is invited to discuss systematically each question with the patient. Whenever the patient gave a wrong answer or ticked the "to be uncertain about the question" option, HCPs are instructed to give the correct answer with background information according to the manual. Then HCPs should ask if the issue is clear or if the patient needs further information. When every question of the KODOA-test has been discussed as described, HCPs should ask the patient if there are any unanswered questions about their DOAC. Although not part of the questionnaire, information about intake time of DOAC was added to cover all instructions needed to counsel. The manual was used by two investigators (CM and VA) during counseling sessions.

\section{Pilot testing}

Comprehension and readability of the finalized KODOA-test was presented to the experts who participated in the focus group discussion and 5 patients who were not included in the validation study. They rated structure, content, comprehension, and response alternatives by writing commentaries in free text. Adaptions were made accordingly.

\section{Validation of the KODOA-test}

\section{Patient eligibility}

Patients were eligible if they filled a prescription for DOAC (rivaroxaban, edoxaban, dabigatran, or apixaban) in a community pharmacy or if a DOAC was present in their medical record, if they suffered from AF, deep vein thrombosis, or pulmonary embolism, if they were $\geq 18$ years old, and if they were able to give written informed consent in German. Patients with an orthopedic indication for DOAC, with dementia, or unable to provide written and/or oral understanding in German were excluded from the study.

\section{Data collection}

A selection of community pharmacists engaged as experts for the state exams of the University of Basel were invited to participate in the research and asked to distribute this invitation to all community pharmacists with whom they were working. Three dedicated community pharmacies located in Basel City and Münchenstein accepted to serve as recruiting places. Pharmacy staff asked patients with a prescription for DOAC to participate in the study and handed out the patient information and the informed consent forms. Patients who agreed to participate fixed 2 appointments within 2 weeks at the community pharmacy.

Patients filled in the KODOA-test 4 times. During the first appointment at the community pharmacy (T1), feasibility and acceptability were also assessed. Immediately after patients filled in the KODOA-test, they rated on a 4-point Likert scale structure, readability and time to fill in the questionnaire, comprehensibility and ambiguity of the items, and the available answer options.

During the second appointment at the community pharmacy, patients filled in the KODOA-test again (T2) and received educational counseling according to the wrong given answers. Immediately after, patients filled the KODOA-test a further time (T2 + edu). Test-retest was assessed with answers from $\mathrm{T} 1$ and $\mathrm{T} 2$, and responsiveness with answers from $\mathrm{T} 2$ and $\mathrm{T} 2+$ edu.

Approximately 2 weeks after T2, patients received the KODOA-test by post (with a paid reply envelope) and filled it at home (T3) to assess sustainability of the educational counseling. Patient follow-up by the treating physician (general practitioner or cardiologist) took place as usual.

Patient characteristics were obtained through written survey at $\mathrm{T} 1$ and included demographic (age, gender, educational background, and duration of DOAC therapy) characteristics and 3 health literacy questions: difficulties to understand written information about medication (yes/neutral/no); difficulties to understand verbal information about medication (yes/neutral/no); confidence when filling out forms (yes/ neutral/no). One question assessed self-estimated knowledge of DOAC with the answer options bad, moderate, good, or excellent. At T3, patients were asked to rate following statement on a five-point Likert scale: "After educational counseling I know more about my DOAC" ( $1=$ I do agree; $5=$ I do not agree).

A personal letter was sent to 29 pharmacists asking them to answer the KODOA-test without consulting additional media and to return it using a prepaid reply envelope.

\section{Statistical methods}

Where appropriate, frequencies, mean, and standard deviations; or median and interquartile ranges (IQR) are presented. Kruskal-Wallis test was used to compare numerical variables 
between 4 groups. All data were entered and analyzed using SPSS statistical package version 24.0 (IBM Corporation, Armonk, NY, USA), and $p$-values $<0.05$ were considered significant.

\section{Validation parameters}

Construct validity was tested with the method of contrasted groups. ${ }^{18}$ Construct validity would be confirmed if the median test score of pharmacists was significantly higher than median test score of patients at T1. Mann-Whitney $U$-test was used to compare numerical data between groups. At T2, patients received educational counseling about DOAC and responsiveness of the KODOA-test was tested. Median test scores before (T2) and after educational counseling (T2 + edu) about DOAC were compared using Wilcoxon test.

The test-retest method was used to demonstrate the stability of the questionnaire over time. Pearson's correlation coefficient was calculated between the test scores from $\mathrm{T} 1$ to T2 and T2 + edu to T3. Additionally, internal consistency or reliability was assessed using Cronbach's $\alpha$, which indicates whether each item of a scale is appropriate for assessing the underlying concept of its scale. Values for Cronbach's $\alpha$ range between 0 and 1; the closer they are to 0 , the less the items are related to one another. Values between 0.5 and 0.7 are the minimal requirement to indicate satisfactory internal consistency. ${ }^{19}$

Index of difficulty and item discrimination ( $D$-value) were calculated to assess performance of single items. The index of difficulty is defined as the proportion of patients answering the item correctly (=number of correct responses/total number of responses). An item with an index of difficulty $>0.75$ is deemed to be too frequently answered correctly. ${ }^{20}$ Item discrimination ( $D$-value) tests how well an item discriminates between people who have a low and high knowledge score. A $D$-value is calculated by subtracting for each item the proportion of respondents answering correctly in the lowest quartile from those answering correctly in the highest quartile, aiming for a mean $D$-value of $50 \%{ }^{20}$

\section{Results}

Out of 45 compiled items, the expert panel selected 15 to be important for knowledge of DOAC. The 15 items were derived from the educational topics "Application and treatment adherence" (5 items, number [nb.] 3, 5-8), "Risk-benefit of treatment" ( 2 items, nb. 2, 4), "Accessing health care providers" (2 items, nb. 12, 13), "Self-care" (2 items, nb. 9, 15), "Relevant blood tests" (1 item nb. 14), "Medication interactions" (2 items, nb. 10, 11), and "Mode of action" (1 item, nb. 1). Two versions of the KODOA-test were developed, 1 for patients with AF and 1 for patients with deep vein thrombosis or pulmonary embolism. The 2 versions differed in the answer options in items 2, 3, and 4, depending on the underlying disease (Figure S1).

Out of 67 invited patients, 32 participated (Table 1). They were prescribed either rivaroxaban $(84.4 \%)$ or apixaban (15.6\%). Mean duration of DOAC use was $1.8 \pm 1.7$ years. Patients had mandatory education $(n=3)$, technical/vocational education $(n=11)$, a higher school certificate $(n=11)$, University degree $(n=4)$, or did not give any information $(n=3)$. A majority of patients stated to have no difficulties to understand written and verbal information about medication and were found to be confident when filling out forms (Table 1). One patient $(3.1 \%)$ was lost to follow-up (T3) and 1 patient did not fill in the knowledge question for follow-up evaluation.

Feasibility was confirmed. Mean time to fill in the KODOA-test was 7.8 \pm 3.0 minutes, and patients were satisfied with the structure, readability, comprehension, and time to fill in the questionnaire (Figure 1).

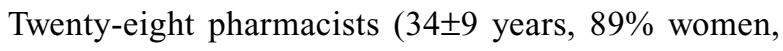
$8 \pm 9$ years working experience) filled in the KODOA-test. Median test score of community pharmacists (13.0 [IQR 1]) was significantly higher compared to test score of patients (10 [IQR 4]) at T1 ( $U$-Test: $p<0.001)$. Median test score of patients at T2 was 11 (IQR 2), and increased significantly to 14 (IQR 3) after educational counseling (Wilcoxon: $p<0.001$ ). Educational counseling about DOAC lasted $14 \pm 7$ minutes. The median number of items whose answers were correct and ticked as confident was calculated. This number increased from 9 (IQR 3) at T2 to 14 (IQR 1) after educational counseling at $\mathrm{T} 2+\mathrm{edu}$ (Wilcoxon: $p<0.001$ ).

Reliability of the KODOA-test was demonstrated. Testretest reliability between $\mathrm{T} 1$ and $\mathrm{T} 2(r=0.800, p<0.001 ; 12$ day mean duration between the tests) and between $\mathrm{T} 2+$ edu and T3 was confirmed $(r=0.644, p<0.001 ; 17$ days mean duration between the tests) (Table 2 ). Internal consistency was acceptable (Cronbach's $\alpha=0.67)$. Cronbach's $\alpha$ was found to increase slightly (0.673-0.678) if items $3,5,6$, or 11 were excluded (Table 3). The index of difficulty for most items was satisfactory $(0.38-0.72)$ (Table 3$)$. The mean $D$-value was $42.5 \%$.

There was no relationship between KODOA-test scores at T1 and patient self-estimated knowledge of DOAC (Table 1). Patients with higher self-estimated knowledge tended to answer with more confidence (Table 1). At T3, all patients agreed that they knew more about their DOAC after having received educational counseling. 
Table I Patient and pharmacists characteristics at TI (baseline)

\begin{tabular}{|c|c|c|c|c|c|}
\hline Characteristics & $\begin{array}{l}\text { Patients } \\
(n=32)\end{array}$ & & & & $\begin{array}{l}\text { Pharmacists } \\
(n=28)\end{array}$ \\
\hline Female (\%) & 65.5 & & & & 89.0 \\
\hline Age (years; mean $\pm S D$ ) & $73 \pm 9$ & & & & $34 \pm 9$ \\
\hline \multicolumn{6}{|l|}{ Indication (\%) } \\
\hline $\mathrm{AF}$ & 62.5 & & & & \\
\hline Deep vein thrombosis/pulmonary embolism & 37.5 & & & & \\
\hline Duration of therapy (years, mean $\pm S D$ ) & $1.8 \pm 1.7$ & & & & \\
\hline \multicolumn{6}{|l|}{ Comprehension of written information about medication (\%) } \\
\hline Without difficulties & 46.8 & & & & \\
\hline Neutral & 21.9 & & & & \\
\hline With difficulties & 31.3 & & & & \\
\hline \multicolumn{6}{|l|}{ Comprehension of verbal information about medication (\%) } \\
\hline Without difficulties & 75.0 & & & & \\
\hline Neutral & 12.5 & & & & \\
\hline With difficulties & 12.5 & & & & \\
\hline \multicolumn{6}{|l|}{ Confidence when filling out forms (\%) } \\
\hline Confidence & 93.8 & & & & \\
\hline No confidence & 6.2 & & & & \\
\hline \multirow[t]{3}{*}{ Self-estimated knowledge (\% of patients [number of patients]) } & Bad & Moderate & Good & Excellent & \\
\hline & 9.4 & 31.3 & 53.0 & 6.3 & \\
\hline & $(n=2)$ & $(n=10)$ & $(n=17)$ & $(n=2)$ & \\
\hline KODOA-test scores (mean \pm SD) & $11.0 \pm 4.4$ & $8.2 \pm 2.4$ & $10.9 \pm 2.1$ & $10.5 \pm 3.5$ & \\
\hline $\begin{array}{l}\text { Number of ticks "being certain about the correctness of the given answer" } \\
\text { (mean } \pm \text { SD) }\end{array}$ & $7.7 \pm 1.5$ & $9.6 \pm 3.4$ & $10.9 \pm 2.6$ & $12.5 \pm 2.1$ & \\
\hline
\end{tabular}

Abbreviations: AF, atrial fibrillation; KODOA, Knowledge Of Direct Oral Anticoagulants; SD, standard deviation.

Time to fill in the questionnaire was acceptable

The questionnaire is clearly and logically structured

The questionnaire is easily readable

The items are easily understandable

The items are specific

Sufficient answer options were available

Agree Somewhat agre

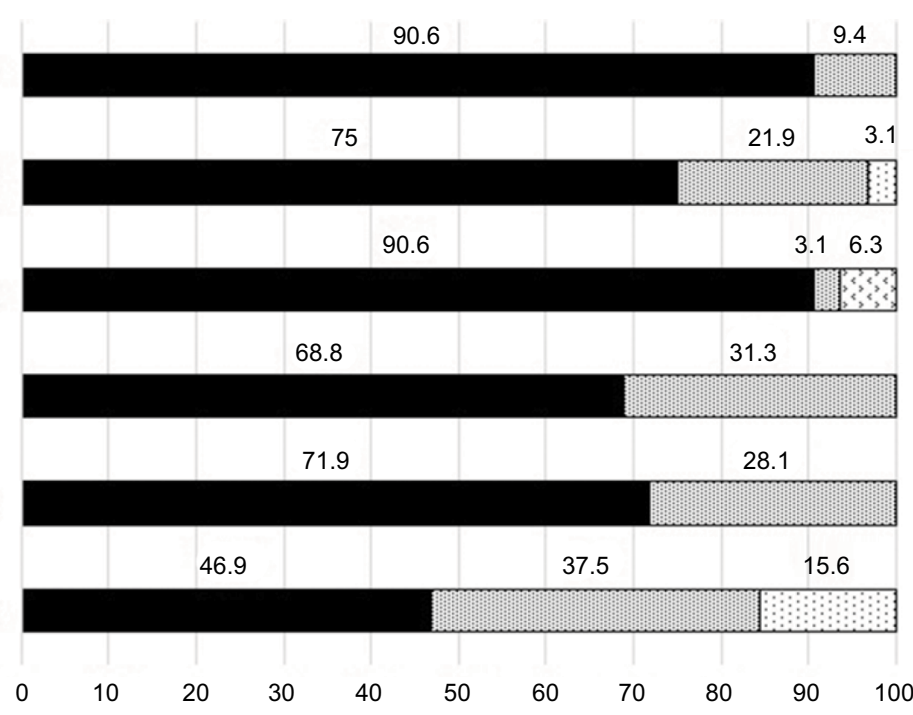

$\square$ Somewhat disagree $\square$ Missing data

Figure I Patients' answers to questions regarding feasibility of the questionnaire $(n=32)$.

\section{Discussion}

The KODOA-test was confirmed to be feasible, comprehensive, reliable, and valid to self-assess patient knowledge of DOAC. Furthermore, the KODOA-test is responsive to change. Content validity was ensured by a developing process with experts of different professions taking care of patients undergoing anticoagulation therapy in their daily practice. Construct validity was supported by significant differences 
Table 2 Mean KODOA-test scores, ie, correctly answered questions ( \pm SD) at TI, T2, T2 + edu (after educational counseling), and T3

\begin{tabular}{llll}
\hline Visit & Patients $(\mathbf{n}=32)$ & Pharmacists $(\mathbf{n}=28)$ & p-value \\
\hline TI & $10.03 \pm 2.7$ & $13.3 \pm 1.0$ & $<0.001$ \\
T2 & $10.06 \pm 2.1^{\mathrm{a}}$ & $\mathrm{NA}$ & \\
$\mathrm{T} 2+$ edu & $13.9 \pm 1.2^{*}$ & $\mathrm{NA}$ & \\
$\mathrm{T}^{\mathrm{b}}$ & $13.1 \pm 1.7$ &
\end{tabular}

Notes: a Mean duration between tests: 12 days. ${ }^{\mathrm{b}} \mathrm{N}=3 \mathrm{I}$ for retest score; mean duration between tests: 17 days. ${ }^{*} p<0.001$.

Abbreviations: KODOA, Knowledge Of Direct Oral Anticoagulants; NA, not applicable; SD, standard deviation; TI, first appointment at the community pharmacy; T2, second appointment at the community pharmacy; T3, 2 weeks after T2.

Table 3 Content and psychometric properties of the KODOA-test items

\begin{tabular}{|c|c|c|c|c|c|}
\hline $\begin{array}{l}\text { Item } \\
\text { number }\end{array}$ & Content & $\begin{array}{l}\text { Patients with } \\
\text { correct } \\
\text { answer (\%) }\end{array}$ & $\begin{array}{l}\text { Index of } \\
\text { difficulty }\end{array}$ & $\begin{array}{l}\text { Item } \\
\text { discrimination }\end{array}$ & $\begin{array}{l}\text { Cronbach's } \alpha \\
\text { if item is } \\
\text { omitted }\end{array}$ \\
\hline I & Name of the DOAC & 93.8 & 0.94 & 18.2 & 0.660 \\
\hline 2 & Duration of therapy & 90.6 & 0.91 & 18.2 & 0.669 \\
\hline 3 & Dosing frequency & 81.3 & 0.81 & 45.5 & 0.673 \\
\hline 4 & Indication for DOAC & 93.8 & 0.94 & 18.2 & 0.656 \\
\hline 5 & What to do if uncertain whether last dose was ingested & 43.8 & 0.44 & 47.7 & 0.673 \\
\hline 6 & What to do in case of a missed dose & 37.5 & 0.38 & 35.2 & 0.678 \\
\hline 7 & What to do in case of double dosing & 37.5 & 0.38 & 56.8 & 0.645 \\
\hline 8 & Does the DOAC work when vomiting immediately after ingestion & 37.5 & 0.38 & 81.8 & 0.640 \\
\hline 9 & Most frequent side effect & 59.4 & 0.59 & 47.7 & 0.647 \\
\hline 10 & Safest OTC analgesic with DOAC & 53.1 & 0.53 & 47.7 & 0.640 \\
\hline II & Whom to ask about safe OTC & 90.6 & 0.91 & 5.7 & 0.673 \\
\hline 12 & When to inform others about DOAC therapy & 87.5 & 0.88 & 27.3 & 0.655 \\
\hline 13 & Recognition of emergencies & 65.6 & 0.66 & 81.8 & 0.605 \\
\hline 14 & How often to visit the doctor for lab monitoring & 59.4 & 0.59 & 51.1 & 0.652 \\
\hline 15 & Carrying an anticoagulation card & 71.9 & 0.72 & 54.5 & 0.638 \\
\hline
\end{tabular}

Abbreviations: DOAC, direct oral anticoagulants; KODOA, Knowledge Of Direct Oral Anticoagulants; OTC, over the counter.

in scores between patients and pharmacists. Finally, the KODOA-test was responsive to educational counseling on DOAC, supporting construct validity.

Patients were able to complete the test within 8 minutes, and rated this time as acceptable. Compared to other assessment questionnaires for knowledge of OAC therapy, the KODOA-test is relatively brief. The AKT and AKA assessment questionnaires need $10-15$ and 20 minutes to be filled in, respectively. ${ }^{11,12}$

The index of difficulty of most items and mean $D$-value was satisfactory. Although 6 items showed a poor level of difficulty, they were retained because they are related to core information about DOAC therapy, such as name, treatment duration, or whom to ask in case of pharmaceutical questions. The KODOA-test showed moderate internal consistency, with a Cronbach's $\alpha$ of 0.67 . The obtained Cronbach's $\alpha$ value is likely a result of the multifactorial nature of the KODOA-test because it consists of items derived from several educational topics. Omission of single items had minimal effect on Cronbach's $\alpha$. The KODOA-test included very easy items and very difficult items. Despite the fact that such grade of difficulty tends to decrease the internal consistency of the scale, ${ }^{21} \mathrm{HCPs}$ need basic questions on the medication used by the patient such as dosing frequency. Finally, a low Cronbach's $\alpha$ might be explained by the shortness of KODOA-test with 15 questions, since internal consistency increases as test length increases..$^{22}$ Therefore, an $\alpha$ of 0.67 seems acceptable.

Stability over time was confirmed by test-retest correlation above the threshold of 0.7 , which indicates adequate reliability. ${ }^{23}$ The mean time between test and retest was 12 days. Recommendations for interval between 2 identical tests vary between 2 days ${ }^{21}$ and 3 months. ${ }^{24}$ Two studies investigating knowledge of anticoagulation with questionnaires had longer intervals of 2-3 months for the retest, ${ }^{10,12}$ and 2 further studies on knowledge of nutrition had intervals of 2 weeks. ${ }^{25,26}$ We selected a short time interval based on ethical considerations, because patients with deficient knowledge should be corrected as soon as possible in order to avoid lifethreatening situations. Knowledge scores remained high after approximately 2 more weeks, demonstrating the sustainability of the educational counseling. In addition, patients agreed uniformly that their knowledge had increased. Nevertheless, 
further follow-ups could be useful to maintain a high level of knowledge. The state of the art currently in Switzerland and in many other countries is silo interventions from several HCPs. It would be best practice to share information and questionnaire results between HCPs.

The majority of patients rated their knowledge of DOAC at baseline as good or excellent. However, this self-estimation did not correspond to KODOA-test scores, suggesting that patients both overestimate and underestimate their knowledge. Consequently, self-estimation of knowledge with a single question should be taken with caution. Further, asking for confidence when answering the item is subject to the same under- and overestimation. However, because of the low number of patients in the bad and excellent self-estimated knowledge groups, this finding needs further investigation.

Interestingly, patients' scores after educational counseling were still below the maximum, with an average of 1.9 erroneous answers. The items concerning safety issues (missed dose, double dosing, vomiting) seem difficult to remember. HCPs should be aware that some pharmacological concepts are more demanding than others.

Previous studies observed an increase of knowledge of OAC therapy following education. ${ }^{27-30}$ However, only 1 study included patients on DOAC, but knowledge assessment methods were not developed for patients on DOAC therapy. ${ }^{27}$ In a recent study, the development of an OAC knowledge questionnaire was reported. ${ }^{12}$ The AKT contains 20 items applicable for DOAC patients and 8 additional items for VKA patients. Overall, the AKT seems to be a valid and reliable questionnaire to measure patient knowledge of oral anticoagulants. However, it is not reported whether the AKT is responsive to change, and scoring seems more complicated as with the KODOA-test. In light of these inconveniences, KODOA-test is likely to be easier to use in patient studies.

Our study has several strengths. First, we followed an evidence-based approach to select items. We coupled a literature search to educational theory to reduce the number of items to the minimum needed to assess knowledge. Second, we used the most recent publication such as the EHRA guidelines to cover exhaustively the characteristics of anticoagulation therapy. Third, we selected experts in all fields of the health care professions to determine the relevant items needed in a self-assessment questionnaire. Fourth, we tested pharmacists as representatives of HCPs. We are confident that similar knowledge results would have been obtained with doctors and nurses. Finally, we developed a manual with answers to obtain standardization of educational counseling.
By doing so, we offer a fast and efficient way of counseling to all HCPs who provide information for patients.

We acknowledge some limitations. First, $52.3 \%$ of the patients refused to participate in the study. Time consumption (ie, 2 visits at the community pharmacy) was likely the reason of this moderate acceptance rate, rather than a low acceptability to answer the KODOA-test. Second, the relatively small number of subjects may limit the ability to generalize results to all patients on DOAC. Importantly, sample size was adequate to show that the KODOA-test is responsive to change and that construct validity is given (data not shown). Third, this study included elderly Swiss German speaking patients with an indication to AF or deep vein thrombosis/pulmonary embolism and taking rivaroxaban or apixaban, that is, only 2 of the 4 commercially available DOACs in Switzerland. Because the KODOA-test targets DOAC knowledge in general, that is, independently of the anticoagulant agent, we cannot think that patients on other anticoagulants (ie, edoxaban or dabigatran) or in another setting (ie, after surgery) would have answered differently. Applicability of the KODOA-test in other settings should nevertheless be investigated in a further study. Finally, the multiple-choice format permits guessing, which may have increased scores in both the patient and pharmacist groups.

\section{Conclusion}

The KODOA-test showed to be valid and reliable in Swiss German speaking elderly outpatients taking DOAC. The application of the KODOA-test in other populations needs confirmation by further research. To our knowledge, the KODOA-test is the first validated questionnaire specific for patients taking DOAC that is responsive to educational counseling. Therefore, the KODOA-test could be used in clinical trials where associations between knowledge of DOAC and adherence or clinical outcomes are of interest.

\section{Acknowledgment}

We would like to thank all experts participating in the development process of the KODOA-test, the 3 community pharmacies for patient recruitment, and all patients and pharmacists for participating in the validation study. We thank Susanne Habegger for her contributions to the literature review and Sonja Luginbühl for the organization of the focus group discussion and KODOA-test piloting. We thank William Caddy for proofreading. The study was developed as an investigator-initiated project by the Pharmaceutical Care Research Group, University of Basel, and received partial 
financial support by Bayer AG. The funders had no role in the design, conduct, analyses, or writing of this study or in the decision to submit for publication.

\section{Disclosure}

The authors report no conflicts of interest in this work.

\section{References}

1. Robertson L, Kesteven P, McCaslin JE. Oral direct thrombin inhibitors or oral factor Xa inhibitors for the treatment of pulmonary embolism. Cochrane Database Syst Rev. 2015;(12):CD010957.

2. Kirchhof P, Benussi S, Kotecha D, et al. 2016 ESC Guidelines for the management of atrial fibrillation developed in collaboration with EACTS. Eur Heart J. 2016;37(38):2893-2962.

3. Olesen JB, Sorensen R, Hansen ML, et al. Non-vitamin K antagonist oral anticoagulation agents in anticoagulant naive atrial fibrillation patients: Danish nationwide descriptive data 2011-2013. Europace. 2015;17(2):187-193.

4. Rodríguez-Bernal CL, Hurtado I, García-Sempere A, Peiró S, SanfélixGimeno G. Oral anticoagulants initiation in patients with atrial fibrillation: real-world data from a population-based cohort. Front Pharmacol. 2017;8:63.

5. Mani H, Lindhoff-Last E. New oral anticoagulants in patients with nonvalvular atrial fibrillation: a review of pharmacokinetics, safety, efficacy, quality of life, and cost effectiveness. Drug Des Devel Ther. 2014;8:789-798.

6. Hernández Madrid A, Potpara TS, Dagres N, et al. Differences in attitude, education, and knowledge about oral anticoagulation therapy among patients with atrial fibrillation in Europe: result of a selfassessment patient survey conducted by the European Heart Rhythm Association. Europace. 2016;18(3):463-467.

7. Wang Y, Kong MC, Lee LH, Ng HJ, Ko Y. Knowledge, satisfaction, and concerns regarding warfarin therapy and their association with warfarin adherence and anticoagulation control. Thrombosis Res. 2014;133(4):550-554.

8. Clarkesmith DE, Pattison HM, Lip GYH, Lane DA. Educational intervention improves anticoagulation control in atrial fibrillation patients: the TREAT randomised trial. PLoS One. 2013;8(9):e 74037.

9. Barcellona D, Contu P, Marongiu F. Patient education and oral anticoagulant therapy. Haematologica. 2002;87(10):1081-1086.

10. Zeolla MM, Brodeur MR, Dominelli A, Haines ST, Allie ND. Development and validation of an instrument to determine patient knowledge: the oral anticoagulation knowledge test. Ann Pharmacother. 2006;40(4):633-638.

11. Briggs AL, Jackson TR, Bruce S, Shapiro NL. The development and performance validation of a tool to assess patient anticoagulation knowledge. Res Social Adm Pharm. 2005;1(1):40-59.

12. Obamiro KO, Chalmers L, Bereznicki LR. Development and validation of an oral anticoagulation knowledge tool (AKT). PLoS One. 2016;11(6):e0158071.
13. Heidbuchel H, Verhamme P, Alings M, et al. Updated European Heart Rhythm Association Practical Guide on the use of non-vitamin K antagonist anticoagulants in patients with non-valvular atrial fibrillation. Europace. 2015;17(10):1467-1507.

14. Lane DA, Wood K. Cardiology patient page. Patient guide for taking the non-vitamin $\mathrm{K}$ antagonist oral anticoagulants for atrial fibrillation. Circulation. 2015;131(16):e412-e415.

15. Wofford JL, Wells MD, Singh S. Best strategies for patient education about anticoagulation with warfarin: a systematic review. BMC Health Serv Res. 2008;8:40.

16. Devellis RF. Scale Development: Theory and Applications. Newbury Park, CA, USA: Sage Publications; 1991.

17. Downing SM. Assessment of knowledge with written test forms. In: Norman GR, van der Vleuten CPM, Newble DI, editors. International Handbook of Research in Medical Education. Vol 2. Dordrecht, The Netherlands: Kluwer Academic Publishers; 2002:647-672.

18. Westen D, Rosenthal R. Quantifying construct validity: two simple measures. J Pers Soc Psychol. 2003;84(3):608-618.

19. Bowling A. Research Methods in Health: Investigation Health and Health Services. 2nd ed. Buckingham, UK: Open University Press; 2002.

20. Anastasi A. Psychological Testing. 6th ed. New York, NY: MacMillan; 1988.

21. Streiner EL, Norman GR. Health Measurement Scales: A Practical Guide to Their Development and Use. Oxford, UK: Oxford University Press; 1989:39-52.

22. Tavakol M, Dennick R. Making sense of Cronbach's alpha. Int J Med Educ. 2011;2:53-55.

23. Paiva CE, Barroso EM, Carneseca EC, et al. A critical analysis of testretest reliability in instrument validation studies of cancer patients under palliative care: a systematic review. BMC Med Res Methodol. 2014;14:8.

24. Nunnally JC. Psychometrie Theory. 2nd ed. New York, NY: McGrawHill; 1978.

25. Jones AM, Lamp C, Neelon M, et al. Reliability and validity of nutrition knowledge questionnaire for adults. J Nutr Educ Behav. 2015;47(1):69-74.

26. Deniz MS, Alsaffar AA. Assessing the validity and reliability of a questionnaire on dietary fibre-related knowledge in a Turkish student population. J Health Popul Nutr. 2013;31(4):497-503.

27. Maikranz V, Siebenhofer A, Ulrich LR, et al. Does a complex intervention increase patient knowledge about oral anticoagulation? A clusterrandomised controlled trial. BMC Fam Pract. 2017;18(1):15.

28. Winans AR, Rudd KM, Triller D. Assessing anticoagulation knowledge in patients new to warfarin therapy. Ann Pharmacother. 2010;44(7-8):1152-1157.

29. Polek C, Hardie T. Warfarin use post hospitalization: pilot comparative effectiveness of telephone follow-up. Rehabil Nurs. 2012;37(2): $80-87$.

30. Vormfelde SV, Abu Abed M, Hua TD, Schneider S, Friede T, Chenot JF. Educating orally anticoagulated patients in drug safety: a cluster-randomized study in general practice. Dtsch Arztebl Int. 2014;111(37):607-614. 


\section{Supplementary materials}

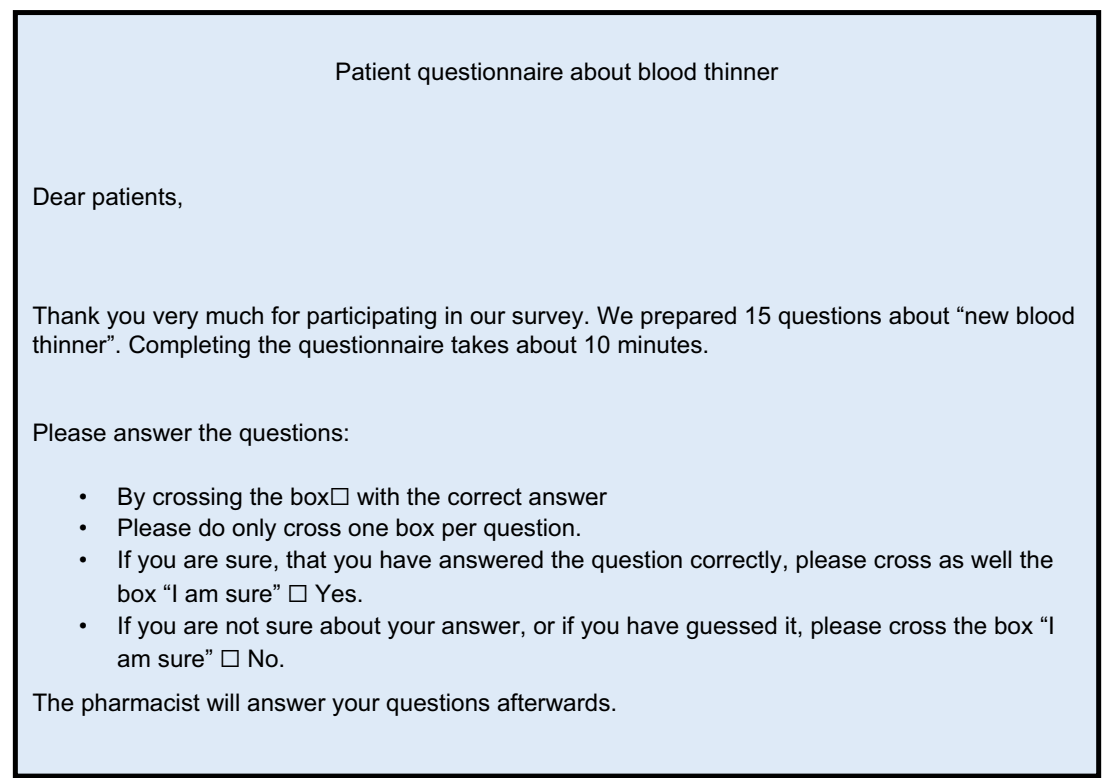

1. What is the name of your blood thinner?

2. How long do you have to take your blood thinner?

3. How often should you take your blood thinner?

4. What does your blood thinner help to prevent you from?

5. Imagine you are uncertain whether you have already taken your blood thinner or not. What are you going to do?

6. Imagine you have forgotten to take the last dose of your medication. What are you going to do?

7. Imagine you have taken double the dose of your tablets/ capsules today by accident. What are you going to do?

8. Imagine you have taken your blood thinner 5 minutes ago and you had to vomit then. Does the blood thinner work less now?

9. Which is a frequent side effect of your blood thinner?

10. Which over the counter painkiller is the safest to take if you are also taking a blood thinner?
11. You would like to take an over the counter medicine, but you do not know if this medicine affects the way your blood thinner works. With whom do you confer this with best?

12. In which situation should you tell your doctor that you are taking a blood thinner?

13. In which situation should you contact your doctor right away or go to the emergency room?

14. How often should you see your doctor for a check-up if you are taking a blood thinner?

15. What should you always carry with you, when you are taking a blood thinner?

Thank you for your participation!

Figure SI Questions of the KODOA-test translated to English (for informative purpose; the English version has not been validated).

\section{Publish your work in this journal}

Drug, Healthcare and Patient Safety is an international, peer-reviewed open access journal exploring patient safety issues in the healthcare continuum from diagnostic and screening interventions through to treatment, drug therapy and surgery. The journal is characterized by the rapid reporting of reviews, original research, clinical, epidemiological and

\section{Dovepress}

post-marketing surveillance studies, risk management, health literacy and educational programs across all areas of healthcare delivery. The manuscript management system is completely online and includes a very quick and fair peer-review system. Visit http://www.dovepress.com/ testimonials.php to read real quotes from published authors. 